\section{Efecto del ultrasonido en la limpieza del sistema de conductos radiculares: revisión de literatura}

\section{Effect of ultrasound on the cleaning of root canal system: a literature review}

\begin{abstract}
Resumen
La limpieza del sistema de conductos radiculares constituye uno de los principales factores para determinar el éxito del tratamiento endodóntico. Por lo tanto, la irrigación complementaria a la instrumentación juega un papel fundamental en la eliminación de las bacterias, restos de dentina y materiales terapéuticos. Además, el método de irrigación es fundamental para garantizar la eficiencia en la limpieza del sistema de conductos radiculares, que puede realizarse manualmente con una jeringa y una aguja, también utilizando dispositivos específicos para esta finalidad, como el ultrasonido. El objetivo de este estudio fue realizar una revisión de literatura sobre el efecto del ultrasonido en la limpieza del sistema de conductos radiculares. Una búsqueda eletrónica fue realizada en las bases de datos PubMed, LILACS y Cochrane Library, con los términos: "passive ultrasonic irrigation", "root canal" y "endodontic irrigation". Los artículos fueron seleccionados a partir de los criterios de inclusión y exclusión. Se concluyó que la irrigación ultrasónica pasiva es eficaz en relación a la eliminación de bacterias, hidróxido de calcio, restos dentinarios y smear layer, utilizándose hipoclorito de sodio $(\mathrm{NaOCl})$ como solución de irrigación, permitiendo así una optimización en la limpieza del sistema de conductos radiculares.
\end{abstract}

Palabras clave: Endodoncia; Ultrasonido; Irrigación; Descontaminación (fuente: DeCS BIREME).

\begin{abstract}
The cleaning of root canal system is one of the principal factors in determining the success of endodontic treatment. Thus, complementary irrigation to the instrumentation plays a fundamental role in the removal of bacteria, dentin remains and therapeutic materials. In addition, the irrigation method is primordial to ensure the cleaning efficiency of the root canal system; it can be performed manually by syringe and needle, but also by using specific devices for this purpose, such as ultrasound. The objective of this study was to perform a review of the literature on the effect of ultrasound on the cleaning of root canal system. An electronic search was performed in the PubMed, LILACS and Cochrane Library databases, with the terms "passive ultrasonic irrigation", "root canal" and "endodontic irrigation". Articles were selected based on the inclusion and exclusion
\end{abstract}

\section{Artículo de Revisión}

\author{
Leandro Jardel da Silva ${ }^{1, a}$, Thaisa Theodoro de Oliveira ${ }^{1, b}$, \\ Andréa Candido dos Reis ${ }^{1, c}$ \\ ${ }^{1}$ Universidade de São Paulo, Faculdade de Odontologia de \\ Ribeirão Preto (FORP-USP), Ribeirão Preto, SP-Brasil. \\ a Especialista en Endodoncia. \\ ${ }^{\mathrm{b}}$ Especialista en Reabilitación Oral. \\ c Doctor en Odontología, área Reabilitación Oral.

\section{Correspondencia:} \\ Andréa Candido Reis \\ Correo electrónico: andreare73@yahoo.com.br \\ Departamento de Materiais Dentários e Prótese, Facul- \\ dade de Odontologia de Ribeirão Preto, Universidade de \\ São Paulo - FORP - USP. Av. do Café, s/n - Monte Alegre \\ - 14040-904
}

\section{Coautores: \\ Leandro Jardel da Silva \\ leandrojasilva@gmail.com \\ Thaisa Theodoro de Oliveira \\ thaisa.oliveira@usp.br}

\section{Editora:}

Sandra Patricia Palomino-Gómez

Universidad Nacional Mayor de San Marcos, Perú.

Conflicto de intereses: los autores declaran no tener conflictos de interés.

\section{Fuente de financiamiento: autofinanciado.}

Recibido: 04/03/2019

Aceptado: 17/06/2019

Publicado: 12/09/2019 
criteria. It was concluded that passive ultrasonic irrigation is effective as the elimination of bacteria, calcium hydroxide, dentin debris and smear layer, using sodium hypochlorite $(\mathrm{NaOCl})$ as an irrigating solution, thus allowing an optimization in the cleaning of root canal system.

Keywords: Endodontics; Ultrasonics; Irrigation; Decontamination (source: $\mathrm{MeSH}$ NLM).

\section{Introducción}

El tratamiento de los conductos radiculares es un procedimiento muy común en Odontologia, que consiste en un método alternativo a la extracción de dientes afectados por caries, pulpitis y necrosis pulpar. Que tiene por objetivo la eliminación de infecciones mediante una limpieza adecuada y la prevención de reinfecciones con un buen sellado de los conductos radiculares ${ }^{1}$.

Aunque todas las fases del tratamiento endodóntico deben realizarse con gran criterio, la limpieza de los conductos radiculares es uno de los principales factores para determinar el éxito del tratamiento endodôntico ${ }^{2}$, porque esta es la responsable de la eliminación de bacterias, tejidos necróticos, medicación intraconducto, restos dentinarios que no son eliminados en la instrumentación, además de funcionar como lubricante para la acción mecánica de la misma ${ }^{3,4}$.

La eficiencia del proceso de irrigacción está diretamente relacionada con la solución irrigante utilizada, así como del método de irrigacción selecionado ${ }^{5}$. Estas soluciones deben tener la habilidad de entrar en contacto con los materiales y estructuras que deben ser eliminados del sistema de conductos radiculares ${ }^{6}$. De esta manera, instrumentos que puedan promover una irrigacción eficiente, así como soluciones irrigantes efectivas han sido ampliamente estudiadas ${ }^{7-15}$.

Existen muchas soluciones irrigantes disponibles en el mercado, como el hipoclorito de sodio, la clorhexidina y las soluciones quelantes, todas con el objetivo de realizar una limpieza efectiva y con propiedades específicas ${ }^{7-15}$.

El método de irrigación también juega un papel fundamental para garantizar la eficiencia de la limpieza del sistema de conductos radiculares, que puede ser realizada manualmente por medio de una jeringa y aguja, pero también utilizando dispositivos como el ultrasonido, EndoVac Irrigation System, RinsEndo ${ }^{\circ}$, entre otros, desarrollados para esta finalidad y disponibles en el mercado. Además, existen estudios que proponen la utlización de técnicas combinadas ${ }^{6,13,16}$.

El uso de equipos para realizar la irrigacción es una discusión bastante cuestionable, ya que la obtención de la limpeza completa y la preparación ideal del sistema de conductos radiculares se torna imposible, dada la complejidad anatómica de los conductos radiculares, irregularidades de las paredes de los conductos como extensiones ovales, istmos y deltas apicales ${ }^{17}$. La literatura demuestra que, en conductos ovales, solo el $40 \%$ de la pared de los conductos puede ser realmente instrumentada en una técnica de instrumentación rotatória ${ }^{6}$.
La introducción del ultrasonido como auxiliar en el tratamiento de los conductos radiculares ha suscitado un gran interés, a pesar de ser controvertido. Aunque no presenta eficiencia comprobada en la etapa de preparación mecánica del sistema de conductos radiculares por no permitir un control adecuado de la instrumentación al someter al diente a perforaciones y desvios radiculares ${ }^{1}$, muchos estudios muestran una eficiência efectiva del ultrasonido cuando se utiliza para fines de limpieza y desinfección de los conductos radiculares en comparación con el método manual ${ }^{7-15}$, ya que el dispositivo ultrasónico convierte la energía eléctrica en ondas ultrasónicas en una determinada frecuencia lo que podría promover algunos efectos biológicos positivos, como la liberación de sustancias ionizantes ${ }^{6}$.

Para aclarar aún más la efectividad y utilización del ultrasonido como una técnica de irrigación en endodoncia, este estudio evalúo por medio de una revisión de literatura, el potencial de limpieza del sistema de conductos radiculares al utilizar esta técnica de irrigación.

\section{Metodología}

Se realizó una búsqueda en las bases de datos PubMed, LILACS y Cochrane Library, con los términos "passive ultrasonic irrigation", "root canal" y "endodontic irrigation". Se encontraron 171 resultados, a partir de estos fue realizada la selección de los trabajos por medio de los criterios de inclusión y exclusión. Los criterios de inclusión fueron: artículos publicados entre los años 2004 al 2019, y que abordaron el uso del ultrasonido en la limpieza del sistema de conductos radiculares. Después de leer los resúmenes, fueron excluídos los artículos que no estaban escritos en inglés y español. De esta manera, fueron seleccionados 29 artículos para lectura y análisis completo, estos se encuentran resumidos en la tabla de acuerdo con los objetivos, grupos experimentales y las conclusiones.

\section{Revisión de la literatura}

Los dispositivos ultrasónicos se introdujeron por primera vez en endodoncia por Richman en $1957^{18}$, donde las limas fueron sometidas a la oscilación de frecuencias ultrasónicas de 25 a $30 \mathrm{kHz}$. Desafortunadamente, se verificó la falta de control del poder de corte de la dentina durante la instrumentación ultrasónica, con resultados en los que fue imposible predecir la forma del conducto radicular preparado, obteniendo perforaciones apicales y formatos irregulares. Por otro lado, cuando se usó el sistema ultrasónico en la etapa de irrigacción del sistema de conductos radiculares, se demostró una buena efectividad para este propósito ${ }^{6}$. 
El uso del sistema ultrasónico como auxiliar en la preparación de los conductos radiculares permite que la irrigación se realice de manera rápida y eficiente, con menos fatiga para el paciente y el profesional. Además, proporciona el aumento de las propiedades de limpieza y desinfección en la instrumentación, cuando las sustancias irrigantes antisépticas son componentes integrales del sistema, permitiendo una acción simultánea ${ }^{19}$.

El sistema ultrasónico, asociado con la irrigacción abundante y continua, también se utiliza para eliminar obstrucciones causadas por cuerpos extraños, como conos de plata y pernos protésicos. Sin embargo, este sistema presenta desventajas tales como, la poca liberación de espacio en la pared lateral del conducto, no proporciona profundidad, presencia de flujo de irrigación deficiente en conductos curvos, posibilidad de desplazamiento de detritos hacia el periápice, y necesidad de capacitación previa del operador ${ }^{6}$.

\section{Tipo de técnica de irrigación ultrasónica}

Dos tipos de irrigación ultrasónica fueron descritas en la literatura, una donde la irrigacción se combinó con la instrumentación ultrasónica simultánea y otra sin instrumentación simultánea, llamada de Irrigación Ultrasónica Pasiva (IUP) ${ }^{1}$.

La técnica de irrigación simultánea a la instrumentación fue menos efectiva en eliminar tejidos y smear layer de los conductos radiculares que la técnica de IUP ${ }^{1}$. Esto se debe a que la lima está en contacto con las paredes del canal y por lo tanto provoca cavitaciones y disminuye la propagación de las corrientes acústicas ${ }^{6}$.

La IUP es realizada mediante la activación ultrasónica de una lima con diámetro menor que el diámetro final de la preparación apical, dentro de un conducto lleno de irrigante, aumentando la eficiencia del desbridamiento y la mayor eliminación de residuos y de smear layer ${ }^{20}$. Dicha irrigación puede realizarse con un flujo de irrigación continuo o intermitente, donde el irrigante se inyecta con una jeringa que se llena varias veces después de cada ciclo de activación ultrasónica, por este método es posible controlar la cantidad de irrigante a través de la profundidad de penetración de la jeringa y volumen administrado. Cuando se utilizan durante tres minutos, ambas técnicas presentan eficacia en la eliminación de residuos dentinarios ${ }^{21}$.

Actualmente el concepto de IUP ha sido cuestionado, algunos reportes indican que el contacto con la pared del conducto radicular ocurre frecuentemente ${ }^{22}$. Sin embargo, la literatura reporta que la IUP presenta buenos resultados para la desinfección de los conductos radiculares, la eliminación de medicación intraconducto, la eliminación del smear layer y barro dentinario 7-14,16,21,23-39.

Irrigación con ultrasonido en el desbridamiento y desinfección del sistema de conductos radiculares

Las lesiones endodónticas se desarrollan en presencia de bacterias, principalmente por especies anaerobias estrictas, siendo el Enterococcus faecalis el principal responsable por el fracaso de los tratamientos endodónticos, porque en comparación con los organismos planctónicos aislados, forman comunidades más resistentes a los antimicrobianos, anticuerpos o a fagocitosis. Por lo tanto, es importante que la técnica de irrigación colabore para la eliminación o reducción de estos microorganismos ${ }^{27,40}$.

La IUP fue eficaz en la eliminación del biofilm del interior de los conductos radiculares, en asociación con hipoclorito de sodio al 2,5\%, en comparación con la irrigación convencional con jeringa ${ }^{23}$. Sin embargo, cuando se asoció con hipoclorito de sodio al 1\%, la IUP no mostró diferencias significativas en la eliminación del biofilm de E. faecalis en comparación con la irrigacción con jeringa ${ }^{9,31}$.

Se observa que el volumen y la velocidad de la solución irrigante son factores que también contribuyen a una mayor eliminación del biofilm de los conductos radiculares ${ }^{23}$.

El tamaño de la jeringa a ser utilizada en la irrigación también es un factor relacionado con el volumen del irrigante, siendo indicado el uso de jeringas de $5 \mathrm{~mL}$, porque asocia la capacidad adecuada con poca dificultad de uso, pues las jeringas con mayor capacidad pueden proporcionar resistencia al movimiento del émbolo, perjudicando el control del procedimiento. Aunque el volumen de la solución es un factor importante, el volumen ideal aún no se ha determinado ${ }^{41}$.

Además, la duración del riego también desempeña un papel importante en la irrigación, ya que juntamente con el suministro del irrigante, influye en el flujo, que se define como la proporción entre el volumen y la duración del irrigante. Otro factor preponderante para la preparación y limpieza de los conductos radiculares es la profundidad de inserción de las agujas y su espesor, siendo necesario que la aguja se inserte de 1 a $2 \mathrm{~mm}$ de la longitud de trabajo, y que la luz de las mismas no sea muy estrecha, porque durante el uso de la solución de hipoclorito de sodio, se cristaliza, haciendo que la irrigación sea impracticable ${ }^{32,41}$.

Con respecto a la irrigación manual, esta no permite un buen control del flujo pues depende de varios factores que pueden variar, incluso con el mismo operador ${ }^{41,42}$.

Otro punto a ser mencionado es el potencial de penetración de la solución irrigante en los túbulos dentinarios, que puede mejorarse mediante las técnicas de irrigación. Se observa que la combinación de la IUP con la irrigación por presión apical negativa promueve la penetración de todo el contenido del irrigante, tanto a nivel apical hasta la longitud de trabajo, como en los conductos laterales, siendo una técnica adecuada para limpiar y desinfectar el sistema de conductos radiculares ${ }^{37}$.

En relación al volumen de irrigación, se demostró que un volumen de aproximadamente $20 \mathrm{~mL}$ de hipoclorito de sodio al 3\% asociado al sistema RinsEndo era suficiente para eliminar E. faecalis, pero no tan eficaz en la IUP; esto puede ser debido al tiempo muy corto de 
irrigación, que fue solo de $20 \mathrm{~s}$, comparado a 3,2 min de irrigación con el sistema RinsEndo. Además, en el tiempo de $45 \mathrm{~s}$ tampoco fue posible eliminar las bacterias del conducto radicular con ninguno de los métodos probados (RinsEndo ${ }^{\circledR}$, IUP e irrigación convencional con jeringa) ${ }^{43}$.

La literatura sugiere que para que haya la eliminación adecuada de las bacterias del sistema de conductos radiculares, la exposición al hipoclorito de sodio debe ser prolongada, ya que el cloro, agente responsable por el efecto antimicrobiano del $\mathrm{NaOCl}$, se consume rápidamente en 2 minutos; sin embargo, aún no existe un consenso general sobre el tiempo de irrigación necesario para eliminar las bacterias intraconducto ${ }^{43}$.

El desbridamiento del conducto radicular con pulpa infectada es otro paso importante para el tratamiento endodóntico y la prevención de infecciones. Se ha demostrado que la irrigación ultrasónica con hipoclorito de sodio al 6\%, asociada a la instrumentación manual y rotatoria, es prometedora, con mejoras en la elminación de biofilm y tejido necrótico ${ }^{10}$.

Además, la IUP tiene la capacidad de elevar la temperatura dentro del canal por encima de $50{ }^{\circ} \mathrm{C}$, promoviendo la activación de la solución irrigante y, en consecuencia, una mayor disolución del tejido ${ }^{7}$.

Irrigación con ultrasonido en la eliminación del smear layer y los restos dentinarios del sistema de conductos radiculares

La limpieza del sistema de conductos radiculares está directamente influenciada por la adecuada eliminación de los residuos y del smear layer, producidos durante la instrumentación del conducto radicular; ya que tales residuos pueden representar una barrera para la acción mecánica y química durante la preparación del conducto radicular ${ }^{28}$.

Además, la capa de smear layer contiene microorganismos que pueden sobrevivir, multiplicarse y penetrar en los túbulos dentinarios, siendo que su permanencia también actúa en la reducción de la eficacia antimicrobiana de los medicamentos intraconducto y una menor capacidad de sellado de los materiales obturadores. Por lo tanto, es extremamente importante su eliminación completa durante el tratamiento endodóntico ${ }^{28}$.

El uso de la irrigación con ultrasonido fue eficaz para la eliminación de los restos dentinarios y el smear layer, en asociación con hipoclorito de sodio al 2\%, presentando mejores resultados en comparación a la irrigación convencional con jeringa ${ }^{12}$.

Se observó una limpieza adecuada con IUP ya sea por flujo intermitente o continuo, así como en la irrigación manual convencional con aguja localizada a $1 \mathrm{~mm}$ de la longitud de trabajo ${ }^{32}$, sin embargo, otros estudios informan que la activación ultrasónica del irrigante hipoclorito de sodio al $2 \%$, combinado con un flujo intermitente produce un efecto acumulativo después de tres ciclos de enfriamiento/activación de la solución irrigante, con una eficiencia mejorada en la eliminación de los residuos dentinarios del interior de los canales radiculares ${ }^{14}$, con acción mejorada en comparación con la irrigación manual, principalmente para la limpieza del tercio apical de los conductos radiculares laterales ${ }^{33}$.

Cuando se compara con otro tipo de irrigación como la irrigación sónica, la IUP presenta mejores resultados en la eliminación de los residuos dentinarios ${ }^{11}$, sin embargo, en conductos rectilíneos el sistema EDDY (irrigación sónica) presenta resultados similares a la IUP para la eliminación de residuos y smear layer ${ }^{28}$.

Otro sistema que tuvo rendimiento similar a la IUP fue el XP endo Finisher, y EndoVac, que eliminaba adecuadamente los desechos del tejido duro, con resultados comparables a los de las técnicas convencionales ${ }^{29,38}$.

Además, se observa que el uso de IUP, independientemente de la solución irrigante asociada, aumenta significativamente la eficacia de la eliminación del smear layer ${ }^{21}$.

\section{Irrigación con ultrasonido y eliminación de hidróxi- do de calcio}

El hidróxido de calcio es ampliamente utilizado como medicación intraconducto entre sesiones debido a sus propiedades regenerativas, antibacterianas, terapéuticas, y a su biocompatíbilidad ${ }^{24}$. Sin embargo, es necesario que el hidróxido de calcio sea totalmente eliminado antes de la obturación permanente del conducto radicular, porque su remanente dificulta la penetración de desinfectantes y el sellado de los túbulos dentinarios y compromete el sellado del sistema de conductos radiculares 24,46 . Por lo tanto, es extremadamente importante que las soluciones irrigantes y las técnicas de irrigación promuevan la eliminación completa de este medicamento.

Solo la irrigación con hipoclorito de sodio no se mostró suficiente para eliminar el hidróxido de calcio, siendo necesario el uso combinado de la eliminación química y mecánica ${ }^{36}$.

Anteriormente, para la eliminación de hidróxido de calcio del sistema de conductos radiculares el método más utilizado era la lima principal o la memoria asociada con la irrigación manual convencional con hipoclorito de sodio y ácido etilenodiaminotetracético (EDTA), sin embargo, estudios indicaron que otras técnicas podrían permitir mejores resultados en la eliminación de este medicamento, como, por ejemplo, el uso del ultrasonido ${ }^{34}$.

La IUP asociada a diversos vehículos tiene la capacidad de ayudar a eliminar los restos de hidróxido de calcio, con resultados superiores en comparación con la irrigación convencional con jeringa ${ }^{8}$.

Cuando los sistemas IUP, EndoActivator y EndoVac se asociaron con instrumentos rotatorios mostraron mejores resultados que la irrigación manual convencional ${ }^{34}$. Sin embargo, la eliminación completa del medicamento es extremadamente difícil y ninguno de los sistemas probados permitió la eliminación completa del medicamento, incluída la IUP 8,34,36,44. 
Otras técnicas de irrigación como EndoVac, EndoActivator, ProUltra, irrigación por aguja biselada, irrigación de doble aguja con ventilación lateral, CanalBrush, XP-endo Finisher, Vibringe e irrigación activada por láser, tampoco fueron capaces de eliminar completamente los restos de la medicación intraconducto. Mientras tanto, el sistema EndoActivator, irrigación activada por láser y el IUP fueron los métodos que permitieron una mayor eliminación del material ${ }^{24,44}$.

El volumen del irrigante y su activación demostraron ser factores importantes para la eliminación efectiva del hidróxido de calcio, siendo que el uso de volúmenes más grandes y la activación con brocha, lima o ultrasonido mostraron menos medicación residual, con mejores resultados en el grupo con asociación de mayor volumen de irrigación e IUP ${ }^{35}$.

\section{Uso de otros sistemas de irrigación en comparación a la irrigación ultrasónica}

La literatura reporta sobre el uso de otros sistemas de irrigación para desinfectar y limpiar el sistema de conductos radiculares, entre ellos el sistema de irrigación Endovac, que se ha utilizado ampliamente y constituye una herramienta importante en el proceso de irrigación del sistema de conductos radiculares. Dicho sistema actúa por presión apical y demuestra resultados superiores en la eliminación del smear layer en comparación con la Irrigación Ultrasónica Pasiva, Irrigación Activada por Láser e irrigación por jeringa y aguja ${ }^{16,30}$. Así como el Sistema EndoActivator, que es un sistema de irrigación sónica que tiene puntas especiales de polímero que son accionadas sónicamente en tres frecuencias diferentes para activar el irrigante ${ }^{11} \mathrm{y}$ también presenta buenos resultados en comparación al IUP y la irrigación activada por láser ${ }^{16}$.

Sin embargo, los estudios muestran, que, aunque todos los sistemas de irrigacción/agitación optimizan la eliminación del smear layer en la región apical del conducto, con los mejores resultados con los sistemas EndoActivator y EndoVac, ninguna de las técnicas (IUP, Endovac u otros usadas) elimina por completo el smear layer en la región apical ${ }^{16,30}$.

Además de estos equipos, el RinsEndo ${ }^{\circledR}$ es un dispositivo recientemente introducido en el mercado y promueve la automatización en la irrigación intraconducto, usando una combinación de irrigación y succión bajo presión hidrodinámica, que proporciona la solución irrigante en el conducto radicular, y la activa automaticamente $(1,5$ $\mathrm{Hz})^{13}$. Este sistema de irrigación mejora la penetración del irrigante en la dentina, permitiendo una mayor eliminación del smear layer, y la reducción de la cantidad de bactérias ${ }^{26}$. Sin embargo, aunque presentaron mejores resultados en comparación con la irrigación con jeringa, presentaron resultados inferiores en la eliminación de los residuos dentinarios, en comparación con la IUP ${ }^{13}$.

Con respecto al efecto bactericida contra E. faecalis; RinsEndo e IUP, cuando son combinados con hipo- clorito de sodio al $1 \%$ + clorhexidina son capaces de eliminar las bacterias del conducto radicular ${ }^{25}$.

De esta manera, observamos que, a pesar de ser considerada una técnica válida para la irrigación del sistema de conductos radiculares, de acuerdo con los estudios analizados, el sistema RinsEndo ${ }^{\oplus}$ presentó resultados similares ${ }^{25,26} \mathrm{o}$ inferiores, en comparación con la IUP ${ }^{13}$.

Además, otra técnica de irrigación que surgió recientemente es la irrigación activada por láser, basada en la vaporización del agua y la formación de burbujas grandes resultantes de la fuerte absorción de la energía del láser en el agua. Durante la oscilación de las burbujas de vapor, la alta presión intraconducto direcciona el fluído hacia afuera del canal, de modo que cuando las burbujas implosionan, se desarrolla una presión negativa y succiona el líquido de vuelta al conducto, induciendo un efecto secundario de cavitación ${ }^{26}$.

La irrigación activada por láser mejora la eliminación del biofilm, el smear layer y los residuos intracanal ${ }^{26,39}$, demostrando ser eficaz en la eliminación de E. faecalis intraconducto de 10 dias, así como en los Sistemas Hidrodinámicos (RinsEndo ${ }^{\circ}$ ) y la IUP ${ }^{26}$.

Por lo tanto, aunque existen muchas otras tantas técnicas disponibles para limpieza y desinfección del sistema de conductos radiculares, la Irrigación Ultrasónica Pasiva juega un papel importante, ya que tiene buenos resultados y es comparable con otras técnicas eficientes.

\section{Soluciones irrigantes asociadas a la Irrigación con ultrasonido}

Actualmente se considera que, la adecuada irrigación y desinfección del sistema de conductos radiculares requiere la combinación de disolventes inorgánicos y orgánicos, con un agente antimicrobiano ${ }^{21}$.

Entre las diversas soluciones utilizadas, el hipoclorito de sodio $(\mathrm{NaOCl})(0,5$ a $6 \%)$ es considerado estándar de oro para la desinfección del sistema de conductos radiculares, lo que contribuye a un tratamiento endodóntico eficaz debido a su capacidad para disolver tejido orgánico, acción bactericida, lubricante y baja toxicidad ${ }^{6}$.

Además del hipoclorito de sodio, otra solución ampliamente utilizada en la irrigación de conductos radiculares es el ácido etilenodiaminotetracético (EDTA) (15 a $17 \%)$, y su uso en conjunto representa un excelente protocolo de irrigación ${ }^{16,21,30,45}$. De esta manera, el $\mathrm{NaOCl}$ ejerce la función de disolvente orgánico y agente antimicrobiano, mientras que el EDTA actúa como disolvente inorgánico o agente de eliminación del smear layer ${ }^{21}$.

Actualmente han surgido otras soluciones irrigantes, como el QMix 2 en 1 (DENTSPLY Tulsa Dental Specialties, Tulsa, OK, EUA), que posee en su composición al ácido poliaminocarboxílico, que actúa como quelante, y la bisbiguanida como agente antimicrobiano, además de un surfactante y agua desionizada ${ }^{2}$. Dicha solución se relacionó con la eliminación del smear layer y de las bacterias como E. faecalis en la irrigación del sistema de conductos radiculares ${ }^{21}$. Así como el uso de clorhexidina 
Tabla 1. Artículos evaluados en la revisión de literatura - objetivos, grupos experimentales y conclusiones

\begin{tabular}{|c|c|c|c|}
\hline & Objetivo del estudio & Grupos experimentales & Conclusión \\
\hline Lee et al. 2004 & $\begin{array}{l}\text { Eliminación de residuos } \\
\text { dentinarios }\end{array}$ & $\begin{array}{ll}- & \text { Irrigación ultrasónica pasiva } \\
& \text { (IUP) } \\
\text { - } & \text { Jeringa }\end{array}$ & IUP más eficaz que la irrigación con jeringa \\
\hline $\begin{array}{l}\text { Burlesson et } \\
\text { al. } 2007\end{array}$ & $\begin{array}{l}\text { Desbridamiento de biofilm y } \\
\text { tejido necrótico }\end{array}$ & $\begin{array}{l}\text { - } \quad \text { Técnica manual y rotatoria } \\
\text { Técnica manual, rotatoria y } \\
\text { ultrasonido }(1 \mathrm{~min})\end{array}$ & $\begin{array}{l}\text { Técnica donde el ultrasonido fue usado presentó } \\
\text { mejores resultados }\end{array}$ \\
\hline $\begin{array}{l}\text { Al-Jadaa et al. } \\
2009\end{array}$ & $\begin{array}{l}\text { Disolución de tejido necrótico } \\
\text { pulpar de los conductos radi- } \\
\text { culares }\end{array}$ & $\begin{array}{ll} & \text { Irrigación pasiva por ultraso- } \\
\text { nido } \\
\text { Localización pasiva del } \\
\text { irrigante }\end{array}$ & $\begin{array}{l}\text { Uso del ultrasonido, eleva la temperatura dentro del } \\
\text { conducto radicular por encima de los } 50^{\circ} \mathrm{C} \text {, promo- } \\
\text { viendo una mayor disolución del tejido.. }\end{array}$ \\
\hline $\begin{array}{l}\text { Jiang et al. } \\
2010\end{array}$ & $\begin{array}{l}\text { Eliminación del residuo } \\
\text { dentinario }\end{array}$ & $\begin{array}{ll} & \text { Activación sónica } \\
\text { - } & \text { Activación ultrasónica }\end{array}$ & $\begin{array}{l}\text { Activación por ultrasonido elimina significativamente } \\
\text { más residuo dentinario que la activación sónica. }\end{array}$ \\
\hline $\begin{array}{l}\text { Balvedi et al. } \\
2010\end{array}$ & $\begin{array}{l}\text { Eliminación del hidróxido } \\
\text { de calcio, mezclando los } \\
\text { diferentes vehículos }\end{array}$ & $\begin{array}{ll}- & \text { Irrigación manual } \\
\text { - } & \text { Irrigación por ultrasonido }\end{array}$ & $\begin{array}{l}\text { Irrigación por ultrasonido presentó menor cantidad de } \\
\text { remanentes de hidróxido de calcio, pero no estadisti- } \\
\text { camente significativo. }\end{array}$ \\
\hline $\begin{array}{l}\text { Bhuva et } \\
\text { al.2010 }\end{array}$ & $\begin{array}{l}\text { Eliminación del biofilm de En- } \\
\text { terococcus faecalis del interior } \\
\text { del conducto radicular. }\end{array}$ & $\begin{array}{ll}\text { - } & \text { Irrigación ultrasónica pasiva } \\
& \text { (IUP) } \\
\text { - } & \text { Irrigación con jeringa } \\
\end{array}$ & $\begin{array}{l}\text { UIP e irrigación con jeringa, no hubo diferencia esta- } \\
\text { dística significativa. }\end{array}$ \\
\hline $\begin{array}{l}\text { Rödig et al. } \\
2010\end{array}$ & $\begin{array}{l}\text { Eliminación del resíduo } \\
\text { dentinario. }\end{array}$ & $\begin{array}{ll}- & \text { RinsEndo® } \\
- & \text { Irrigación a través de jeringa } \\
\text { Irrigación ultrasónica pasiva } \\
\text { (IUP) }\end{array}$ & $\begin{array}{l}\text { IUP eliminó los residuos dentinarios significativamen- } \\
\text { te mejor que RinsEndo®, que a su vez, fue mejor que } \\
\text { la irrigación manual con jeringa. }\end{array}$ \\
\hline $\begin{array}{l}\text { van der Sluis } \\
\text { et al. } 2010\end{array}$ & $\begin{array}{l}\text { Eliminación del residuo } \\
\text { dentinario del interior de los } \\
\text { conductos radiculares }\end{array}$ & - $\quad$ Activación ultrasónica & $\begin{array}{l}\text { El hipoclorito de sodio más eficiente como irrigante } \\
\text { durante la activación ultrasónica. }\end{array}$ \\
\hline $\begin{array}{l}\text { Spoorty et } \\
\text { al.2013 }\end{array}$ & $\begin{array}{l}\text { Eficacia de la penetración de } \\
\text { irrigantes en la longitud de } \\
\text { trabajo y conductos laterales }\end{array}$ & $\begin{array}{ll}\text { - } & \text { Irrigación ultrasónica pasiva } \\
& \text { (IUP) } \\
\text { - } & \text { Irrigación convencional por } \\
& \text { aguja } \\
\text { - } & \text { Irrigación por presión apical } \\
& \text { negativa (IPAN) } \\
\text { - IUP + IPAN }\end{array}$ & $\begin{array}{l}\text { IUP + IPAN fue el único grupo capaz de alcanzar la } \\
\text { penetración de la solución irrigante de contraste tanto } \\
\text { en la longitud de trabajo como en los canales laterales. }\end{array}$ \\
\hline $\begin{array}{l}\text { Chachovan et } \\
\text { al. } 2013\end{array}$ & $\begin{array}{l}\text { Efecto bactericida de dos } \\
\text { sistemas }\end{array}$ & $\begin{array}{ll}- & \text { RinsEndo } \\
\text { - } & \text { Irrigación ultrasónica pasiva } \\
& \text { (IUP) } \\
\text { - } & \text { Enjuague manual } \\
\end{array}$ & $\begin{array}{l}\text { Sistema hidrodinámico y IUP, cuando combinados } \\
\text { con hipoclorito de sodio al } 1 \% \text { + clorhexidina son capa- } \\
\text { ces de eliminar bacterias del conducto radicular. }\end{array}$ \\
\hline $\begin{array}{l}\text { Bago et al. } \\
2014\end{array}$ & $\begin{array}{l}\text { Efectividad en la eliminación } \\
\text { de E. faecalis intraconducto }\end{array}$ & $\begin{array}{ll}\text { - } & \text { RinsEndo® } \\
\text { - } & \text { Irrigación a través de jeringa } \\
\text { irrigación activada por láser }\end{array}$ & $\begin{array}{l}\text { Concluyeron que todas las técnicas, excepto la irriga- } \\
\text { ción manual son igualmente eficaces en la eliminación } \\
\text { de E. faecalis intraconducto de } 10 \text { dias. }\end{array}$ \\
\hline $\begin{array}{l}\text { Chavez-An- } \\
\text { drade et al. } \\
2014\end{array}$ & $\begin{array}{l}\text { Eficacia en la limpieza de los } \\
\text { conductos radiculares }\end{array}$ & $\begin{array}{ll}\text { - } & \text { Irrigación ultrasónica pasiva } \\
\text { (IUP) flujo continuo } \\
\text { - } \quad \text { Irrigación ultrasónica pasiva } \\
\text { (IUP) flujo intermitente } \\
\text { Irrigación manual convencio- } \\
\text { nal (IMC) }\end{array}$ & $\begin{array}{l}\text { IUP y IMC promueven una limpieza similar de los } \\
\text { conductos radiculares principal y laterales en el tercio } \\
\text { medio. PUHIUP con flujo intermitente es más eficaz } \\
\text { para la limpieza de los conductos laterales en el tercio } \\
\text { apical. }\end{array}$ \\
\hline $\begin{array}{l}\text { Ordinola-Za- } \\
\text { pata et al. } 2014\end{array}$ & $\begin{array}{l}\text { Evaluar la eliminación de bio- } \\
\text { film en conductol radicular }\end{array}$ & $\begin{array}{ll}\text { - } & \text { Irrigación con aguja } \\
\text { - } & \text { Endoactivator } \\
& \text { Irrigación ultrasónica pasiva } \\
\text { - } & \text { IUP) } \\
\end{array}$ & $\begin{array}{l}\text { Irrigación activada por láser con hipoclorito de sodio } \\
\text { al } 6 \% \text { mejoró significativamente la limpieza de la den- } \\
\text { tina infectada con biofilm, seguida por la IUP. }\end{array}$ \\
\hline $\begin{array}{l}\text { Faria et al. } \\
2014\end{array}$ & $\begin{array}{l}\text { Eliminación del hidróxido } \\
\text { de calcio del sistema de con- } \\
\text { ductos radiculares a través de } \\
\text { instrumentación rotatoria aso- } \\
\text { ciada a sistema de irrigación }\end{array}$ & $\begin{array}{ll}\text { - } & \text { EndoActivator } \\
\text { - } & \text { EndoVac } \\
& \text { Irrigación ultrasónica pasiva } \\
& \text { (IUP) } \\
\text { - } & \text { Irrigación convencional con } \\
& \text { aguja (IMC) }\end{array}$ & $\begin{array}{l}\text { Instrumento rotatorio combinado con EndoActiva- } \\
\text { tor, EndoVac y IUP se mostró más eficiente cuando } \\
\text { comparado al instrumento rotatorio combinado con } \\
\text { el IMC }\end{array}$ \\
\hline $\begin{array}{l}\text { Freire et al. } \\
2015\end{array}$ & $\begin{array}{l}\text { Eficacia de la irrigación en la } \\
\text { eliminación de residuos de } \\
\text { tejidos duros y calidad del } \\
\text { relleno del canal radicular }\end{array}$ & $\begin{array}{ll}\text { - } & \text { Irrigación ultrasónica pasiva } \\
& \text { (PUI) } \\
\text { - } & \text { EndoVac }\end{array}$ & $\begin{array}{l}\text { El PUI y Endovac fueron igualmente eficientes en la } \\
\text { eliminación de restos de tejido duro y la calidad del } \\
\text { relleno del conducto radicular fue semejante en los dos } \\
\text { grupos, sin influencia del método de irrigación. }\end{array}$ \\
\hline $\begin{array}{l}\text { Guerreiro-Ta- } \\
\text { nomaru et al. } \\
2015\end{array}$ & $\begin{array}{l}\text { Efectividad en la eliminación } \\
\text { de E. faecalis intraconducto }\end{array}$ & $\begin{array}{ll}\text { - } & \text { Irrigación ultrasónica pasiva } \\
& \text { (IUP) } \\
\text { - } & \text { Irrigación manual convencio- } \\
& \text { nal (IMC) } \\
\text { - } & \text { Sin irrigación (control). } \\
\end{array}$ & $\begin{array}{l}\text { IUP y IMC con } \mathrm{NaOCl} \text { al } 1 \% \text { contribuyen para la des- } \\
\text { infección, pero son incapaces de eliminar E. faecalis del } \\
\text { sistema de conductos radiculares. }\end{array}$ \\
\hline $\begin{array}{l}\text { Alturaiki et al. } \\
2015\end{array}$ & $\begin{array}{l}\text { Eficacia de sistemas de } \\
\text { irrigación en la eliminación de } \\
\text { hidróxido de calcio }\left(\mathrm{Ca}[\mathrm{OH}]_{2}\right) \\
\text { del conducto radicular }\end{array}$ & $\begin{array}{ll}- & \text { Lima maestra apical } \\
\text { - } & \text { EndoVac } \\
\text { - } & \text { EndoActivator } \\
& \text { ProUltra }\end{array}$ & $\begin{array}{l}\text { Ninguna de las técnicas investigadas eliminó comple- } \\
\text { tamente el } \mathrm{Ca}(\mathrm{OH})_{2} \text {. El sistema EndoActivator fue el } \\
\text { mejor sistema en comparación con las demás técnicas. }\end{array}$ \\
\hline
\end{tabular}




\begin{tabular}{|c|c|c|c|}
\hline & Objetivo del estudio & Grupos experimentales & Conclusión \\
\hline Joy et al. 2015 & $\begin{array}{l}\text { Eficiencia de la irrigación en } \\
\text { la eliminación del biofilm } \\
\text { bacteriano }\end{array}$ & $\begin{array}{ll} & \text { Irrigación estática } \\
\text { - } & \text { Irrigación ultrasónica pasiva } \\
\text { (IUP) }\end{array}$ & $\begin{array}{l}\text { Irrigación ultrasónica pasiva contribuye para la elimi- } \\
\text { nación del biofilm bacteriano }\end{array}$ \\
\hline $\begin{array}{l}\text { Cherian et al. } \\
2016\end{array}$ & $\begin{array}{l}\text { Evaluar la acción antimicro- } \\
\text { biana de los irrigantes y téc- } \\
\text { nica de irrigación ultrasónica } \\
\text { pasiva }\end{array}$ & $\begin{array}{l}\text { Irrigación ultrasónica pasiva (IUP) } \\
2 \% \text { de clorhexidina } \\
\text { Irrigación ultrasónica pasiva (IUP) } \\
0,1 \% \text { de dicloridrato de octenidina }\end{array}$ & $\begin{array}{l}\text { Los autores reportan que la irrigación ultrasónica pasi- } \\
\text { va mejora la acción antimicrobiana de los irrigantes }\end{array}$ \\
\hline $\begin{array}{l}\text { Toljan et al. } \\
2016\end{array}$ & $\begin{array}{l}\text { Evaluar eficacia antimicro- } \\
\text { biana de tres técnicas de } \\
\text { irrigación después del uso de } \\
\text { volumen estandarizado de } \\
\mathrm{NaOCl} \text { y con tiempo e irriga- } \\
\text { ción estandarizados. }\end{array}$ & 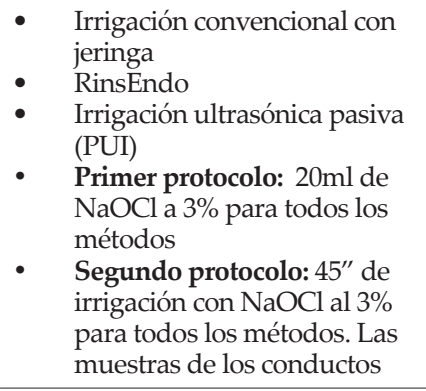 & $\begin{array}{l}\text { RinsEndo fue más efectivo que el PUI con volumen } \\
\text { estandarizado ( } 20 \mathrm{~mL} \text { ). Sin embargo, el RinsEndo } \\
\text { proporcionó mayor reducción bacteriana en ambos } \\
\text { protocolos cuando se utilizó la menor cantidad del irri- } \\
\text { gante y proporcionó mayor tiempo de contacto. }\end{array}$ \\
\hline $\begin{array}{l}\text { Tanomaru-Fil- } \\
\text { ho et al. } 2016\end{array}$ & $\begin{array}{l}\text { Eficacia en la limpieza del } \\
\text { sistema de conductos radi- } \\
\text { culares }\end{array}$ & $\begin{array}{ll}\text { - } & \text { Irrigación ultrasónica pasiva } \\
\text { (IUP) flujo intermitente } \\
\text { - Irrigación ultrasónica pasiva } \\
\text { (IUP) flujo contínuo } \\
\text { Irrigación manual convencio- } \\
\text { nal (IMC) }\end{array}$ & $\begin{array}{l}\text { PUI con flujo intermitente o continuo y el IMC con } \\
\text { la aguja colocada a } 1 \mathrm{~mm} \text { de longitud de trabajo } \\
\text { presentaron una limpieza eficiente de los conductos } \\
\text { radiculares. }\end{array}$ \\
\hline $\begin{array}{l}\text { Zorzin et al. } \\
2016\end{array}$ & $\begin{array}{l}\text { Evaluar eliminación de } \\
\text { hidróxido de calcio a través } \\
\text { de irrigación con diferentes } \\
\text { volúmenes y métodos de } \\
\text { activación. }\end{array}$ & $\begin{array}{l}\text { Irrigación convencional con } \\
\text { diferentes volúmenes ( } 0 \mathrm{~mL} \text {, } \\
0,5 \mathrm{~mL}, 1 \mathrm{~mL}, 2 \mathrm{~mL}, 4 \mathrm{~mL} \text { ou } \\
8 \mathrm{~mL}) \\
\text { Activación mecánica con } 2 \mathrm{o} \\
4 \mathrm{~mL} \text { a través de lima, brocha } \\
\text { (CanalBrush) o irrigación } \\
\text { ultrasónica pasiva (IUP) }\end{array}$ & $\begin{array}{l}\text { Ningún procedimiento de irrigación fue capaz de eli- } \\
\text { minar completamente el } \mathrm{Ca}(\mathrm{OH})_{2} \text {. IUP fue el método } \\
\text { de activación más eficaz y la irrigación con volumen } \\
\text { de } 8 \mathrm{~mL} \text { fue la más efectiva. }\end{array}$ \\
\hline $\begin{array}{l}\text { Pabel et al. } \\
2017\end{array}$ & $\begin{array}{l}\text { Evaluar la eliminación de } \\
\text { hidróxido de calcio de con- } \\
\text { ductos radiculares rectos. }\end{array}$ & $\begin{array}{ll}\text { - } & \text { Irrigación ultrasónica pasiva } \\
& \text { (IUP) } \\
\text { - } & \text { RinsEndo } \\
\text { - } & \text { EndoActivator } \\
\text { - } & \text { CanalBrush } \\
\text { - } & \text { Irrigación manual con jeringa }\end{array}$ & $\begin{array}{l}\text { No fue posible obtener la eliminación completa } \\
\text { del hidróxido de calcio del conducto radicular por } \\
\text { ninguna de las técnicas investigadas, sin embargo, el } \\
\text { más alto grado de limpeza fue obtenido por el uso de } \\
\text { ultrasonido. }\end{array}$ \\
\hline $\begin{array}{l}\text { Koçak et al } \\
2017\end{array}$ & $\begin{array}{l}\text { Eficacia de la eliminación del } \\
\text { smear layer }\end{array}$ & $\begin{array}{l}\text { - irrigación ultrasónica pasiva } \\
\text { (IUP) asociado a diversas } \\
\text { soluciones irrigantes }\end{array}$ & $\begin{array}{l}\text { Independiente de la solución irrigante esta técnica } \\
\text { (IUP) aumentó significativamente la eficacia de la } \\
\text { eliminación del smear layer }\end{array}$ \\
\hline $\begin{array}{l}\text { Urban et al. } \\
2017\end{array}$ & $\begin{array}{l}\text { Evaluaron comparativamente } \\
\text { el uso de irrigación manual, } \\
\text { sónica y ultrasónica en la } \\
\text { eliminación del smear layer }\end{array}$ & $\begin{array}{ll}- & \text { Sistema sónica (EDDY) } \\
\text { - } & \text { Irrigación ultrasónica pasiva } \\
\text { (IUP) } & \text { Irrigación manual } \\
\end{array}$ & $\begin{array}{l}\text { EDDY y IUP tuvieron desempeño significativamente } \\
\text { superior a la irrigación manual en la eliminación de } \\
\text { residuos y el smear layer, en conductos radiculares } \\
\text { rectilíneos. }\end{array}$ \\
\hline $\begin{array}{l}\text { Leone et al. } \\
2017\end{array}$ & $\begin{array}{l}\text { Reducción de restos de tejido } \\
\text { duro acumulados en el con- } \\
\text { ducto radicular }\end{array}$ & $\begin{array}{ll}\text { - } & \text { Presión positiva apical, } \\
\text { - } & \text { Irrigación ultrasónica pasiva } \\
& \text { (IUP) } \\
\text { - } \quad \text { Self-adjusting File } \\
\text { - XP endo-Finisher. }\end{array}$ & $\begin{array}{l}\text { IUP y XP endo Finisher tuvieron mejores resultados en } \\
\text { la eliminación de residuos de tejido duro. }\end{array}$ \\
\hline $\begin{array}{l}\text { Gokturk et al. } \\
2017\end{array}$ & $\begin{array}{l}\text { Capacidad de eliminar } \\
\text { hidróxido de calcio }\end{array}$ & $\begin{array}{ll} & \text { Irrigación aguja biselada } \\
\text { - } & \text { Irrigación doble aguja con } \\
& \text { ventilación lateral } \\
\text { - } & \text { CanalBrush } \\
\text { - } & \text { XP-endo Finisher } \\
\text { - } & \text { Vibringe } \\
& \text { Irrigación ultrasónica pasiva } \\
\text { - } & \text { IUP) } \\
\end{array}$ & $\begin{array}{l}\text { La irrigación activada por láser y IUP fueron los méto- } \\
\text { dos que permitieron mayor elminación de material. }\end{array}$ \\
\hline $\begin{array}{l}\text { Karade et al. } \\
2017\end{array}$ & $\begin{array}{l}\text { Eliminación del smear layer } \\
\text { intraconducto }\end{array}$ & $\begin{array}{ll}\text { - } & \text { Irrigación jeringa y aguja } \\
\text { - } & \text { Irrigación sónica } \\
& \text { Irrigación ultrasónica pasiva } \\
& \text { (IUP) } \\
\text { - } & \text { EndoVac. }\end{array}$ & $\begin{array}{l}\text { Sistema EndoVac demostró resultados significati- } \\
\text { vamente mejores cuando comparado a los demás } \\
\text { sistemas evaluados. }\end{array}$ \\
\hline $\begin{array}{l}\text { Mancini et al. } \\
2018\end{array}$ & $\begin{array}{l}\text { Eficacia de diversos métodos } \\
\text { de irrigación en la eliminación } \\
\text { del smear layer. }\end{array}$ & $\begin{array}{ll}\text { - } & \text { EndoActivator } \\
\text { - } & \text { Irrigación ultrasónica pasiva } \\
& \text { (IUP) } \\
\text { - } & \text { Endovac } \\
\text { - } & \text { Irrigación activada por láser }\end{array}$ & $\begin{array}{l}\text { EndoActivator y EndoVac obtuvieron mejores resul- } \\
\text { tados. } \\
\text { Sistemas de irrigación/agitación mejoran la elimina- } \\
\text { ción del smear layer. }\end{array}$ \\
\hline
\end{tabular}


(CHX) $(0,2 \text { a } 2 \%)^{25,27}$, que constituye un irrigante utilizado por su acción antimicrobiana y su sustantividad, que tiene como desventaja la no disolución de tejidos, una característica importante para la desinfección efectiva del sistema de conductos radiculares.

Otra solución que ha surgido recientemente es el diclorhidrato de octenidina (OCT) (Octenisept, Schulke e Mayr $\mathrm{GmBH}$, Norderstedt, Alemanha) al $0,1 \%$, que, aunque poco estudiada, muestra resultados positivos, con una mejora en su acción antimicrobiana cuando se asocia con la IUP ${ }^{27}$.

\section{Conclusión}

La revisión de literatura ha demostrado que la irrigacción ultrasónica pasiva es eficaz para eliminar microrganismos, eliminar hidróxido de calcio, residuos dentinarios y el smear layer, y para aumentar la acción de la solución irrigante, permitiendo así, una optimización en la limpieza del sistema de conductos radiculares.

\section{Referencias bibliográficas}

1. Pedrazzi V, De Oliveira-Neto JM, Sequeira P, Fedorowicz Z, Nasser M. Hand and ultrasonic instrumentation for orthograde root canal treatment of permanent teeth. J Appl Oral Sci. 2010;18(3):268-72.

2. Haapasalo M, Shen Y, Wang Z, Gao Y. Irrigation in endodontics. Br Dent J. 2014;216(6):299-303.

3. Castelo-Baz P, Varela-Patino P, Cantatore G, Dominguez-Perez A, Ruiz-Pinon M, Miguens-Vila R, et al. In vitro comparison of passive and continuous ultrasonic irrigation in curved root canals. J Clin Exp Dent. 2016;8(4):e437-e441

4. Chen S, Liu J, Dong G, Peng B, Yang P, Chen Z, et al. Comparison between ultrasonic irrigation and syringe irrigation in clinical and laboratory studies. J Oral Sci. 2016;58(3):373-8.

5. Plotino G, Pameijer CH, Grande NM, Somma F. Ultrasonics in endodontics: a review of the literature. J Endod. 2007;33(2):81-95.

6. van der Sluis LWM, Versluis M, Wu MK, Wesselink PR. Passive ultrasonic irrigation of the root canal: a review of the literature. Int Endod J. 2007;40(6):415-26.

7. Al-Jadaa A, Paqué F, Attin T, Zehnder M. Necrotic pulp tissue dissolution by passive ultrasonic irrigation in simulated accessory canals: impact of canal location and angulation. Int Endod J. 2009;42(1):59-65.

8. Balvedi RPA, Versiani MA, Manna FF, Biffi JCG. A comparison of two techniques for the removal of calcium hydroxide from root canals. Int Endod J. 2010;43(9):763-8.

9. Bhuva B, Patel S, Wilson R, Niazi S, Beighton D, Mannocci $F$. The effectiveness of passive ultrasonic irrigation on intraradicular Enterococcus faecalis biofilms in extracted single-rooted human teeth. Int Endod J. 2010;43(3):241-50.

10. Burleson A, Nusstein J, Reader A, Beck M. The in vivo evaluation of hand/rotary/ultrasound instrumentation in necrotic, human mandibular molars. J Endod. 2007;33(7):782-7.
11. Jiang L-M, Verhaagen B, Versluis M, van der Sluis LWM. Evaluation of a sonic device designed to activate irrigant in the root canal. J Endod. 2010;36(1):143-6.

12. Lee S-J, Wu M-K, Wesselink PR. The effectiveness of syringe irrigation and ultrasonics to remove debris from simulated irregularities within prepared root canal walls. Int Endod J. 2004;37(10):672-8.

13. Rödig T, Sedghi M, Konietschke F, Lange K, Ziebolz D, Hülsmann M. Efficacy of syringe irrigation, RinsEndo and passive ultrasonic irrigation in removing debris from irregularities in root canals with different apical sizes. Int Endod J. 2010;43(7):581-9.

14. Van der Sluis LWM, Vogels MPJM, Verhaagen B, Macedo R, Wesselink PR. Study on the influence of refreshment/activation cycles and irrigants on mechanical cleaning efficiency during ultrasonic activation of the irrigant. J Endod. 2010;36(4):737-40.

15. Zehnder M. Root Canal Irrigants. J Endod. 2006;32(5):389-98.

16. Mancini M, Cerroni L, Iorio L, Dall'Asta L, Cianconi L. FESEM evaluation of smear layer removal using different irrigant activation methods (EndoActivator, EndoVac, PUI and LAI). An in vitro study. Clin Oral Investig. 2018;22(2):993-9.

17. Vivan RR, Duque JA, Alcalde MP, Só MVR, Bramante CM, Duarte MAH. Evaluation of Different Passive Ultrasonic Irrigation Protocols on the Removal of Dentinal Debris from Artificial Grooves. Braz Dent J. 2016;27(5):568-72.

18. Richman MJ. The use of ultrasonics in root canal therapy and root resection. J Med. 1957;12:12-8.

19. Mozo S, Llena C, Forner L. Review of ultrasonic irrigation in endodontics: increasing action of irrigating solutions. Med Oral Patol Oral Cir Bucal. 2012;17(3):e512-6.

20. Cidade FH, Schmidt TF, Dos Santos LGP, Tay FR, da Silveira Teixeira C, Felippe MCS, et al. Effect of passive ultrasonic irrigation on diffusion of hydroxyl ion through radicular dentine. Clin Oral Investig. 2016;20(2):247-52.

21. Koçak S, Bağcı N, Çiçek E, Türker SA, Can Sağlam B, Koçak MM. Influence of passive ultrasonic irrigation on the efficiency of various irrigation solutions in removing smear layer: a scanning electron microscope study. Microsc Res Tech. 2017;80(5):537-42.

22. Boutsioukis C, Tzimpoulas N. Uncontrolled Removal of Dentin during In Vitro Ultrasonic Irrigant Activation. J Endod. 2016;42(2):289-93.

23. Joy J, Mathias J, Sagir VMM, Babu BP, Chirayath KJ, Hameed H. Bacterial Biofilm Removal Using Static and Passive Ultrasonic Irrigation. J Int Oral Health. 2015;7(7):42-7.

24. Gokturk H, Ozkocak I, Buyukgebiz F, Demir O. Effectiveness of various irrigation protocols for the removal of calcium hydroxide from artificial standardized grooves. J Appl Oral Sci. 2017;25(3):290-8.

25. Cachovan G, Schiffner U, Altenhof S, Guentsch A, Pfister W, Eick S. Comparative antibacterial efficacies of hydrodynamic and ultrasonic irrigation systems in vitro. J Endod. 2013;39(9):1171-5. 
26. Bago Jurič I, Plečko V, Anić I. Antimicrobial efficacy of Er,Cr:YSGG laser-activated irrigation compared with passive ultrasonic irrigation and $\operatorname{RinsEndo}\left({ }^{\oplus}\right)$ against intracanal Enterococcus faecalis. Photomed Laser Surg. 2014;32(11):600-5.

27. Cherian B, Gehlot PM, Manjunath MK. Comparison of the Antimicrobial Efficacy of Octenidine Dihydrochloride and Chlorhexidine with and Without Passive Ultrasonic Irrigation - An Invitro Study. J Clin Diagn Res. 2016;10(6):ZC71-7.

28. Urban K, Donnermeyer D, Schäfer E, Bürklein S. Canal cleanliness using different irrigation activation systems: a SEM evaluation. Clin Oral Investig. 2017;21(9):2681-7.

29. Leoni GB, Versiani MA, Silva-Sousa YT, Bruniera JFB, Pécora JD, Sousa-Neto MD. Ex vivo evaluation of four final irrigation protocols on the removal of hard-tissue debris from the mesial root canal system of mandibular first molars. Int Endod J. 2017;50(4):398-406.

30. Karade P, Chopade R, Patil S, Hoshing U, Rao M, Rane $\mathrm{N}$, et al. Efficiency of Different Endodontic Irrigation and Activation Systems in Removal of the Smear Layer: A Scanning Electron Microscopy Study. Iran Endod J. 2017;12(4):414-8.

31. Guerreiro-Tanomaru JM, Chávez-Andrade GM, de Faria-Júnior NB, Watanabe E, Tanomaru-Filho M. Effect of Passive Ultrasonic Irrigation on Enterococcus faecalis from Root Canals: An Ex Vivo Study. Braz Dent J. 2015;26(4):342-6.

32. Tanomaru-FIlho M, Torres FFE, Chávez-Andrade GM, Miano LM, Guerreiro-Tanomaru JM. Intermittent or continuous ultrasonically activated irrigation: micro-computed tomographic evaluation of root canal system cleaning [Internet]. Clin Oral Invest. 2016;20:1541-6.

33. Chávez-Andrade GM, Guerreiro-Tanomaru JM, Miano LM, de Toledo Leonardo R, Tanomaru-Filho M. Radiographic evaluation of root canal cleaning, main and laterals, using different methods of final irrigation [Internet] Rev Odontol UNESP. 2014;43(3):333-7.

34. Faria G, Viola KS, Kuga MC, Garcia AJA, Daher VB, De Pasquali Leonardo MF, et al. Effect of rotary instrument associated with different irrigation techniques on removing calcium hydroxide dressing. Microsc Res Tech. 2014;77(8):642-6.

35. Zorzin J, Wießner J, Wießner T, Lohbauer U, Petschelt A, Ebert J. Removal of Radioactively Marked Calcium Hydroxide from the Root Canal: Influence of Volume of Irrigation and Activation. J Endod. 2016;42(4):637-40.
36. Pabel A-K, Hülsmann M. Comparison of different techniques for removal of calcium hydroxide from straight root canals: an in vitro study [Internet]. Odontology. 2017; 105:453-9.

37. Spoorthy E, Velmurugan N, Ballal S, Nandini S. Comparison of irrigant penetration up to working length and into simulated lateral canals using various irrigating techniques [Internet]. Int Endod J. 2013;46:815-22.

38. Freire LG, Iglecias EF, Cunha RS, Dos Santos M, Gavini G. Micro-Computed Tomographic Evaluation of Hard Tissue Debris Removal after Different Irrigation Methods and Its Influence on the Filling of Curved Canals. J Endod. 2015;41(10):1660-6.

39. Ordinola-Zapata R, Bramante CM, Aprecio RM, Handysides R, Jaramillo DE. Biofilm removal by $6 \%$ sodium hypochlorite activated by different irrigation techniques. Int Endod J. 2014;47(7):659-66.

40. Dioguardi M, Di Gioia G, Illuzzi G, Laneve E, Cocco A, Troiano G. Endodontic irrigants: Different methods to improve efficacy and related problems. Eur J Dent. 2018;12(3):459-66.

41. Boutsioukis C, Lambrianidis T, Kastrinakis E, Bekiaroglou P. Measurement of pressure and flow rates during irrigation of a root canal ex vivo with three endodontic needles. Int Endod J. 2007;40(7):504-13.

42. Moreno D, Conde AJ, Lorońo G, Adorno CG, Estevez R, Cisneros R. Comparison of the Volume of Root Canal Irrigant Collected by 2 Negative Pressure Needles at Different Flow Rates of Delivery. J Endod. 2018;44(5):838-41.

43. Toljan I, Bago I, Jurič, Anić I. Eradication of Intracanal Enterococcus Faecalis Biofilm by Passive Ultrasonic Irrigation and RinsEndo System. Acta Stomatol Croat. 2016;50(1):14-22.

44. Alturaiki S, Lamphon H, Edrees H, Ahlquist M. Efficacy of 3 different irrigation systems on removal of calcium hydroxide from the root canal: a scanning electron microscopic study. J Endod. 2015;41(1):97-101.

45. Siu C, Baumgartner JC. Comparison of the debridement efficacy of the EndoVac irrigation system and conventional needle root canal irrigation in vivo. J Endod. 2010;36(11):1782-5.

46. Silva LJM, Pessoa OF, Teixeira MBG, Gouveia CH, Braga RR. Micro-CT evaluation of calcium hydroxide removal through passive ultrasonic irrigation associated with or without an additional instrument. Int Endod J. 2015;48(8):768-73. 
